\title{
e-Phaïstos
}

e-Phaïstos

Revue d'histoire des techniques / Journal of the history

of technology

VI-2 2017 | 2018

La diversité du patrimoine technique africain

\section{Modes et techniques d'exploitation des arbres chez les Toupouri du Nord-Cameroun}

Forest Management by the Tupuri in Northern Cameroon

Jean Gormo

\section{OpenEdition}

Journals

Édition électronique

URL : http://journals.openedition.org/ephaistos/3475

DOI : 10.4000/ephaistos.3475

ISSN : 2552-0741

Éditeur

IHMC - Institut d'histoire moderne et contemporaine (UMR 8066)

\section{Référence électronique}

Jean Gormo, « Modes et techniques d'exploitation des arbres chez les Toupouri du Nord-Cameroun », e-Phaïstos [En ligne], VI-2 2017 | 2018, mis en ligne le 16 novembre 2018, consulté le 10 décembre 2020. URL : http://journals.openedition.org/ephaistos/3475; DOI : https://doi.org/10.4000/ephaistos. 3475

Ce document a été généré automatiquement le 10 décembre 2020.

Tous droits réservés 


\title{
Modes et techniques d'exploitation des arbres chez les Toupouri du Nord-Cameroun
}

\author{
Forest Management by the Tupuri in Northern Cameroon
}

Jean GORMO

1 Établis dans un environnement difficile, les Toupouri du Nord-Cameroun ont, dans ce milieu contraignant marqué par l'aridité et par les aléas climatiques importants, su adapter leur manière de vivre et leur savoir-faire. En effet, l'homme ne se place pas en simple observateur du monde animal et végétal mais est susceptible de l'anthropiser, de façon temporaire ou irréversible ${ }^{1}$ Ainsi, ce peuple du Nord-Cameroun possède des connaissances approfondies des propriétés des feuilles, fruits et bois présents sur son territoire. Ces matières végétales et les savoir-faire techniques associés sont présents dans de nombreuses activités socio-économiques.

\begin{abstract}
«La connaissance des usages traditionnels des plantes sauvages est un apport à la pénétration de la mentalité d'une population; de même que l'histoire de la flore économique dont elle dispose et des techniques qu'elle met en jeu pour en tirer profit est intimement liée à son histoire sociale et culturelle $»^{2}$
\end{abstract}

2 C'est dans cette démarche que nous nous inscrivons et nous allons donc, dans cet article, exposer la place de l'exploitation des arbres dans la société toupouri.

3 L'arbre a toujours joué un rôle polyvalent dans la vie des sociétés rurales du NordCameroun. L'article analyse non seulement les divers cheminements techniques que suivaient les Toupouri pour exploiter leur environnement végétal, mais s'intéresse également aux multiples utilisations et transformations réservées aux arbres. Il s'agit ici de mettre en exergue les techniques utilisées dans l'architecture chez les Toupouri, et notamment celles relatives à la toiture des cases et aux greniers. 


\section{Quête et choix des arbres : une diversité de modalités techniques}

\section{Une découverte fortuite}

4 À la question de savoir à quand remonte l'identification des atouts d'un arbre, les Toupouri du Nord-Cameroun, répondent unanimement qu'ils ont acquis cette technique de leurs parents. C'est-à-dire que l'histoire orale, sans donner de date, rapporte que c'est la répétition régulière et la transmission des pratiques dans le cercle familial qui a inscrit ces savoirs dans un héritage commun.

5 Les artisans et les sculpteurs de la région s'accordent à dire que les atouts d'un bois peuvent être découverts de manière fortuite grâce à un concours de circonstances. Par la suite, ils peuvent divulguer cette découverte aux autres artisans, ratifiant ainsi un choix qui deviendra traditionnel. La transmission de ce savoir se fait à la fois de manière orale et pratique. Lors des différents échanges qui ont souvent cours entre les techniciens du bois, les découvertes individuelles sont présentées aux uns et aux autres qui les enregistrent pour les mettre en pratique dès que l'occasion se présente.

6 Par ailleurs, les artisans reconnaissent que la crise actuelle des essences végétales ${ }^{3}$ détermine régulièrement l'affectation de nouvelles plantes ligneuses à des activités précises. À titre d'exemple, un artisan toupouri cite le Ficus dubulbula qu'il découvrit fortuitement lorsqu'il se trouva confronté à la rareté du Prosopis africana traditionnellement affecté à la fabrication des mortiers. Confronté à cette crise, il choisit le Ficus dubulbula, qui offre un bois mi-dur avec une durabilité naturelle moyenne. Ce choix fut fait en regard des ressemblances morphologiques de ces essences, sans toutefois prendre en compte leurs qualités technologiques propres ${ }^{4}$. Cependant, ce nouveau choix ne garantit pas toujours les avantages exclusifs du Prosopis africana.

7 À la suite de ces découvertes, les principes sont établis et deviennent avec le temps des éléments de référence en termes de matériaux de travail du bois. C'est de cette manière que les Toupouri du Nord-Cameroun ont reconnu et reconnaissent encore les qualités mécaniques et technologiques de leurs arbres. Cette démarche est aussi valable dans les Grassfields du Cameroun, où Nizesété note :

« qu'un arbre quelconque, abattu sans aucune connaissance préalable de ses caractéristiques physiques, mécaniques ou esthétiques, peut être ensuite apprécié puis adopté si à l'issue de sa mise en œuvre, il donne un résultat satisfaisant dans le domaine d'activité où il est mis en application. Dès lors l'essence concernée est classée, son choix entériné et sa sélection perpétuée $»^{5}$.

8 D'autres méthodes sont utilisées par les Toupouri pour détecter les qualités physiques et mécaniques d'une essence végétale.

\section{L'observation et l'habitude}

9 À la question de savoir comment, dans un environnement végétal dense et hétérogène, les Toupouri ont reconnu les qualités mécaniques et technologiques d'un arbre, la réponse suivante apporte des précisions: «Le paysan peut reconnaitre les qualités 
mécaniques et technologiques d'un arbre, arbuste à partir de ses capacités à résister face aux agents destructeurs notamment les feux de brousse, les intempéries et les termites xylophages. Il peut aussi juger ces qualités à partir de l'effort qu'il fournit lors de l'abattage d'un arbre. $»^{6}$. Ces qualités sont recherchées pour la fabrication des outils soumis à une forte expression d'usage comme le mortier, le pilon et pour les supports de greniers ou de hangars.

Sous l'action du vent, certains arbres et arbustes perdent leurs branches ou sont tout simplement déracinés. Dans cette zone au climat essentiellement sec, ces branches et ces troncs d'arbres sont soumis à l'action des intempéries et, de ce fait, leur vitesse de décomposition est vite évaluée. Ceux des troncs et branches qui se putréfient rapidement sont reconnus de mauvaise qualité pour certaines activités. Cependant, ces arbres peuvent être bien appréciés dans d'autres secteurs d'activité qui ne nécessitent pas de résistance. D'autres qualités peuvent être recherchées : l'absence des nœuds dans le bois, la rectitude du tronc. Ce sont bien ces qualités que recherchent les Mangbetu lorsqu'ils veulent réaliser un bon tambour :

«Compte tenu de la grande taille du futur tambour du chef, l'arbre doit avoir un diamètre précis à mi-hauteur car ce n'est que dans cette portion du tronc que les fibres du bois conviennent ; plus près des racines, le bois devient humide et les fibres grossières ; plus près du houppier, le bois devient cassant et les fibres trop menues. Les nœuds dans le bois s'opposent à une propagation homogène des vibrations et il faudra inspecter l'écorce à la hauteur choisie pour bien vérifier qu'il n'y ait pas eu de branche latérale dans cette portion de tronc. Par contact, l'écorce épouse les fibres externes du tronc et sa structure donne une image de leur rectitude, de leur fermeté, de leur cohésion. Simplement en regardant les troncs, Mabondane peut « prévoir » le tambour à l'intérieur du fût... À l'aide du plat d'une cognée, du manche d'une herminette et d'un bâton lourd, Mabondane va systématiquement percuter les troncs à hauteur d'homme et écouter la réponse sonore de l'arbre $»^{7}$.

11 Par ailleurs les feux de brousse réguliers et généralement allumés en saison sèche pour des besoins d'agriculture ou d'élevage, représentent également un facteur d'évaluation qui permet d'avoir une idée des diverses caractéristiques d'une essence. En effet, une plante ligneuse qui se consume facilement donne la preuve de sa mollesse. Il en est de même des essences végétales qui ne produisent pas de bons charbons de bois (charbon qui se transforme rapidement en cendre) ou celles dont les troncs se laissent ronger facilement par les termites. C'est le cas de Bombax costatum, Calotropis procera, Annona senegalensis et Adansonia digitata qui sont des arbres aux bois légers, tendres, qui présentent une mauvaise durabilité naturelle. Ces essences végétales sont sollicitées dans la fabrication des tam-tams qui requièrent des bois légers et tendres, faciles à creuser et à transporter.

12 Par contre, les arbres dont les bois résistent aux assauts des feux de brousse (l'écorce seule étant attaquée) et des termites fournissent de ce fait la preuve de leur dureté. Plusieurs essences végétales possèdent ces caractéristiques, notamment Tamarindus indica (Ba'aré en langue vernaculaire toupouri), Prosopis africana (Wa'a), Ximenia americana (Booré), Afzelia africana (Fèw) et Guiera senegalensis (Furii).

13 Les bûcherons, à défaut des précédentes méthodes, optent pour une autre à travers laquelle ils agissent sur le végétal. Par habitude et par expérience, ces derniers peuvent tester les capacités de résistance et de durabilité naturelle des ligneux. Ils le font souvent 
lors de l'abattage des arbres. Généralement, lorsque les bûcherons fournissent beaucoup d'efforts pour abattre un arbre alors que leur hache est bien aiguisée, ils reconnaissent d'emblée une espèce ligneuse au bois dur et lourd. L'abattage des arbres obéit à certains procédés que nous allons analyser dans la partie qui suit.

\section{L'abattage des arbres au Nord-Cameroun}

14 Dans les sociétés africaines en général et chez les Toupouri en particulier, le végétal a toujours été la première source d'approvisionnement en matière première. Il se prête à de multiples usages. Par ailleurs, au regard de leur polyvalence fonctionnelle, les arbres se sont imposés dans la pensée collective comme des forces extra-humaines dont l'abattage passe nécessairement par l'observation d'un rituel bien précis. Il existe chez les Toupouri des forêts sacrées qui constituent le lieu par excellence du culte rendu aux ancêtres. La pérennité de ces forêts est garantie par l'existence de nombreux interdits qui entourent leur gestion.

\section{Les rites d'abattage}

15 Avant de parler des rites d'abattage proprement dits, il convient de s'interroger sur les fondements qui les ont longtemps sous-tendus. Il est unanimement admis que, du fait de la perméabilité de la mémoire collective, le recensement exhaustif de ces fondements reste difficile à établir. Ainsi, pour essayer d'apporter une lumière sur ce pan important de l'histoire de ce peuple, il est judicieux d'énoncer certains faits qui peuvent élucider ce phénomène.

Les rites d'abattage découleraient du principe selon lequel l'arbre serait assimilé à un être se rapprochant de l'humain. Dans la culture toupouri, l'arbre possède tout comme l'homme un souffle divin. Ils sont tous deux issus de la même matrice divine. Les sociétés africaines ont longtemps considéré l'arbre ainsi et ses éléments morphologiques sont comparés à ceux de l'être humain. Les expressions utilisées pour désigner les parties de l'arbre ont une valeur dénominative très importante. Que ce soit chez les Toupouri, Mofou, Foulbé ou Guiziga du Nord-Cameroun, les branches sont assimilées aux bras/ mains (Doo ko'o en Toupouri, Djoudé lekki en Foulfouldé, Mahang en Guiziga et Hawal en Mofou). La sève est assimilée au sang (Tchii ko'o) et les feuilles aux oreilles (Souk ko'o). Ainsi, certains arbres sont considérés comme des entités protectrices et les Toupouri utilisent Anogeissus leiocarpus (Sèw) comme poteau-fétiche - planté à l'entrée de chaque concession - censé protéger toute la maison.

17 D'autres pratiques des peuples du Nord-Cameroun démontrant le caractère anthropomorphe des arbres sont connues. Il existe dans le répertoire oral, des contes qui la mettent en exergue. Les relations qui existent entre l'homme et le végétal sont donc très fortes dans cette culture. Ces rapports inextricables sont symbolisés par l'existence des forêts sacrées et par les rites qui entourent l'abattage des arbres ${ }^{8}$. Aussi comprend-on aisément pourquoi la coupe sauvage d'un arbre a toujours été considérée comme un crime dans la société toupouri. Un tel caractère sacré de l'arbre ne se cantonne pas à l'Afrique et peut se retrouver dans d'autres sociétés. Ainsi, dans la même veine, Elsdon Best, cité par Lévy-Bruhl, précise que : 
«Le Maori d'autrefois, dit M. Elsdon Best, regardait les arbres avec de tout autres yeux que nous. Quand le Maori entrait dans une forêt, il se sentait au milieu de ses proches: car l'homme et les arbres n'avaient-ils pas une origine commune, étant l'un et les autres les descendants de Tane? Donc il se trouvait, pour ainsi dire, parmi ses parents, et cette forêt avait un principe de vie tapu (sacré), exactement comme l'homme en a un »9. présentent au pied de l'arbre à abattre. Conformément aux prescriptions du devin, le maître des cérémonies sacrifie l'animal demandé. Tout en psalmodiant, il asperge l'arbre avec le sang de l'animal. Le sacrifice a une finalité essentiellement propitiatoire. Les prières se résument en ces termes : « Arbre, je te donne ce sacrifice afin que par son sang, nous soyons purifiés de notre acte. Puisse aucun malheur nous arriver. Esprit de l'arbre, libère-nous la voie. $»^{11}$. Le sacrifice offert a pour objectif de demander pardon à l'esprit de l'arbre et le prier de garantir une bonne réalisation des travaux. Ce sacrifice propitiatoire est attesté également au Vanuatu où :

"Sur l'île de Tanna comme dans les autres îles de TAFEA, l'abattage d'un arbre à pirogue est accompagné d'une succession de rites. Ceux-ci sont destinés à implorer les esprits bénéfiques pour favoriser le bon déroulement de l'opération.

A Aniwa, la fabrication des pirogues est l'apanage exclusif d'un clan, spécialisé dans ce travail. D’après nos informations, une action préliminaire consiste à offrir un poulet et un laplap à la personne chargée de construire la pirogue. Cette pratique constitue à la fois un premier paiement pour le travail et en même temps une demande d'autorisation pour abattre l'arbre qui sera utilisé $»^{12}$.

21 Généralement, le maitre initiateur est contraint de préciser la raison de l'abattage ; car l'abattage d'un arbre doit répondre à une exigence noble dont la valeur socioculturelle est unanimement reconnue (fabrication des tambours, architecture, etc...). Ce rituel est formellement prescrit pour les grands arbres. Lors de cette incantation, le maitre initiateur est tenu de glorifier l'arbre à abattre, de faire son éloge. Cet hymne incantatoire rend compte de la valorisation religieuse et sociale de l'arbre. Les incantations prononcées sont en fait des prières dont le but est de supplier les esprits de l'arbre de quitter leur demeure pour aller s'abriter dans d'autres arbres proches. Sans ce rituel, l'arbre dans sa chute pourrait entraîner le bûcheron dans son sillage et, de plus, l'essence abattue ne donnerait plus satisfaction dans l'emploi envisagé. Par exemple, lorsque l'essence est destinée à fabriquer un tambour, on peut entendre chez les Toupouri du

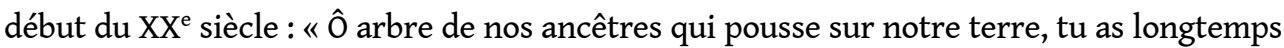
satisfait les désirs de nos aïeux, tu es bon pour les tambours rituels, au nom de la coutume 
je te coupe. $»^{13}$ Au regard du caractère essentiellement oral de la culture toupouri, la parole prend une dimension très importante dans le rituel. Elle déborde le cadre de la simple communication pour entrer dans la sphère ontologique. Elle est expressive et consacre l'accomplissement du rituel.

Cette cérémonie rituelle qui précède l'abattage des arbres au Nord-Cameroun est semblable à celle qui se déroulait chez les tambourineurs du Burundi où l'abattage d'un arbre pour la fabrication d'un tambour donnait lieu à une grande festivité. Dans ce pays, les coups de hache des bûcherons étaient scandés par les rythmes des tambours et des chants $^{14}$.

Nizesété, en décrivant ce rituel chez les peuples des Grassfields du Cameroun, remarque :

«Ce rituel dépasse cependant le cadre restreint du Cameroun car, on le retrouve en Europe notamment chez les paysans roumains, autrichiens ou allemands. Alain Bourras (1989) raconte à propos : «dans certaines parties de l'Autriche, de vieux paysans croient encore que les arbres des forêts sont animés, ils ne permettent pas qu'on leur fasse une entaille dans l'écorce sans raison spéciale. Leurs pères leur ont enseigné que l'arbre sent la blessure tout comme un homme. En abattant un arbre, ils lui demandent pardon. On dit aussi que dans le Haut-Palatinat (Allemagne), les vieux bûcherons demandent en secret à un bel arbre vigoureux avant de l'abattre, de leur pardonner leur geste $» »^{15}$.

\section{Les outils d'abattage}

Pour l'abattage des arbres et arbustes, les bûcherons utilisent des haches et des machettes traditionnelles. Ces outils sont pour la plupart fabriqués localement par les forgerons. La hache leur est indispensable pour la coupe des troncs et la machette sert principalement à l'ébranchage. Il faut remarquer ici que l'utilisation de ces outils est prééminente et remonte à plusieurs siècles chez les Toupouri. La caste des forgerons toupouri, les mougouri, continue actuellement son activité de forge en pays toupouri. Ils ne sont pour autant pas les uniques pourvoyeurs des outils servant à l'abattage ; l'utilisation des cordes est aussi courante pour faciliter la chute de l'arbre.

\section{Les techniques d'abattage}

L'abattage des grands arbres est de manière générale un travail d'équipe. Cette étape dure plusieurs jours. L'abattage de l'arbre requiert une méthode propre et nécessite beaucoup de précision. La première phase consiste à préparer la zone d'abattage. Les responsables de l'abattage délimitent préalablement la zone et s'assurent que personne ne se trouve à l'intérieur. Pour ce travail, ils doivent éliminer les chicots ou les brindilles qui peuvent provoquer des rebonds. Dès que cela est fait, ils nettoient la base de l'arbre en défrichant la broussaille et les branches de la zone d'abattage. Par la suite, il est nécessaire de dégager la voie sur au moins trois mètres. Enfin, ils doivent élaguer à l'aide d'une machette appelée lorgo'o le tronc de l'arbre à abattre pour éviter toute fissure lors de la chute.

Ces modalités techniques préparatoires achevées, l'équipe d'abattage peut commencer le travail proprement dit. L'orientation de la chute de l'arbre dans la direction choisie n'est pas hasardeuse et tient compte d'un certain nombre de mesures très précises. Sous le 
regard vigilant du maitre initiateur, les consignes sont données à l'abatteur. De manière générale, l'entaille de direction est nécessaire pour tous les arbres aux diamètres importants. Sa profondeur doit correspondre au tiers du diamètre de l'arbre à abattre. L'entaille de direction étant faite, les initiés peuvent commencer l'exécution du trait d'abattage qui se situe sur le côté opposé de l'entaille. Une fois le trait d'abattage effectué, l'arbre entame normalement sa chute. C'est alors que l'abatteur s'éloigne dans la voie de retraite qu'il a préalablement aménagée.

\section{Le moment de l'abattage}

$\mathrm{Au} \mathrm{XIX}^{\mathrm{e}}$ siècle encore, le moment et surtout la saison d'abattage des arbres étaient prescrits par la coutume. Ainsi, pour abattre les arbres, les Toupouri se remettaient à la tradition qui prévoyait ce travail en saison sèche. Le choix de cette saison obéissait à plusieurs raisons. Elle correspondait à la fin des travaux champêtres et au début des activités secondaires, notamment le renouvellement de la couverture des cases. C'est également la période pendant laquelle la paille nécessaire aux toitures est disponible. Les activités liées au bois et même au divertissement se pratiquent souvent en saison sèche. Pendant cette saison, les déplacements sont fluides et les populations disposent d'assez de temps pour se consacrer à d'autres activités que l'agriculture, qui est prééminente en saison des pluies. C'est d'ailleurs le moment choisi pour pratiquer le gourna ${ }^{16}$.

Sur le plan quotidien, la coupe des arbres se déroule généralement le matin afin d'éviter d'affronter les heures les plus chaudes du jour en raison de la rudesse du climat. L'abattage d'un arbre peut s'étendre sur plusieurs jours; cela dépend de la nature de l'arbre à abattre et de son diamètre. Une fois l'arbre abattu, quelle en est la finalité ?

\section{Les utilisations du bois}

De par sa polyvalence fonctionnelle, le bois constitue le support matériel de plusieurs activités notamment pour l'architecture, le mobilier, les instruments de musique, les outils de chasse, pêche, agriculture, et sert même comme combustible. Jean-Louis Parmentier le qualifie de: «La plus ancienne ressource naturelle de l'homme. Il lui a fourni : chauffage, outils, nourriture, et abri tout au long de son interminable voyage à travers les âges ${ }^{17}$. Dans le cadre de cet article, l'accent est mis sur l'architecture qui regroupe la fabrication de la charpente et la construction des greniers.

\section{Charpente en bois}

La construction de la case commence par le déblayage du terrain et son nivelage. Par la suite, le paysan trace un cercle à l'aide d'une ficelle préalablement attachée à un piquet planté au sol. Tout dépend du diamètre de la case à construire. Plus la case est grande, plus la ficelle doit être longue. Il peut alors creuser le sol sur une profondeur d'environ 60 $\mathrm{cm}$ à l'aide d'une pioche. Après cette opération, la mise en place de la fondation commence et est suivie de l'élévation du mur, dont l'argile est mélangée à certaines herbes utilisées comme dégraissants. Il s'agit notamment de Combretum aculeatum ( Dambéllé) et Andropogon pseudapricus (Dramgui). basée sur le travail de la paille. La confection des toitures se fait à l'aide de la paille et des 
cordes. La toiture a une forme conique, une base ronde et un sommet pointu. Utilisateur averti des graminées suivantes : Andropogon tectorum (Ganebo), Echinochlon pyramidalis (Laï ), Hyparrhenia sp. (Hompai), le paysan toupouri maîtrise le lieu et le moment de cueillette de celles-ci. Le renouvellement des toits de chaume se déroule en saison sèche, période pendant laquelle l'herbe est disponible.

À la fin des travaux champêtres, les paysans tressent la paille à l'ombre des grands arbres $\mathrm{du}$ village. Pour ce faire, ils sollicitent une gamme variée de plantes utilisées comme cordes. Il s'agit principalement de l'écorce de Bauhinia thonningii (Buuri), Acacia hebecladoïdes (Girlin), Sterculia setigera (Gasuri). Ces cordes servent non seulement à tresser la paille, mais aussi à attacher le bois de charpente.

La fin des travaux champêtres coïncide avec le début de la saison sèche, période pendant laquelle la coupe des arbres se déroule puisque ceux-ci sont hors sève. Les bois sont rassemblés entre les murs des cases jusqu'à leur séchage. Jamais les bois humides ne sont utilisés dans la charpente car à leur séchage, ils peuvent se déformer et rendre fragile l'ensemble de la structure.

34 La charpente, constituée de bois liés à un ensemble de ceintures circulaires, représente l'ossature de la toiture. Une charpente est généralement faite de sept ceintures circulaires qui vont de la base au sommet. Squelette de la toiture, la charpente se doit d'être solide. Les bourrelets circulaires horizontaux fixés à intervalle régulier de 40 à $50 \mathrm{~cm}$ renforcent la rigidité de celle-ci. Cette solidité lui est nécessaire pour lutter contre les intempéries. Dans une région où profilèrent termites et insectes xylophages, les bois qui constituent la charpente doivent présenter de bonnes caractéristiques mécaniques. Conscients de cette situation, les Toupouri ont opéré des choix judicieux dans une gamme variée d'essences végétales. Ainsi, les bois sollicités pour la charpente doivent être de bonne qualité (milourd, lourd, dur ou mi-dur), suffisamment longs, rectilignes, sains et présenter une bonne durabilité naturelle. Les enquêtes de terrain révèlent l'emploi de Terminalia macroptera (Hobé), Gardenia embescens (Baw), Combretum aculeatum (Dambéllé), Acacia hebecladoïdes (Girlin) et Anogeissus leiocarpus (Sew) chez les Toupouri. La distinction entre bois dur et bois léger influence leur utilisation dans l'architecture.

Sur la crête du mur, un autre boudin soigneusement serré et fixé est posé et attaché généralement à la charpente. Il constitue l'assise du toit et sa fixation dépend de la résistance de celui-ci aux coups de vent et aux tornades récurrents dans la région. Ce boudin et ces bourrelets circulaires sont obtenus à partir des branchages de Guiera senegalensis (Furri).

Après la construction de la charpente, les paysans la recouvrent de paille. Pour rendre la toiture résistante aux assauts des vents, les Toupouri fixent la paille à la charpente à l'aide d'une corde solide. Le toit est souvent fabriqué au sol et ensuite transporté puis fixé sur le couronnement du mur. 


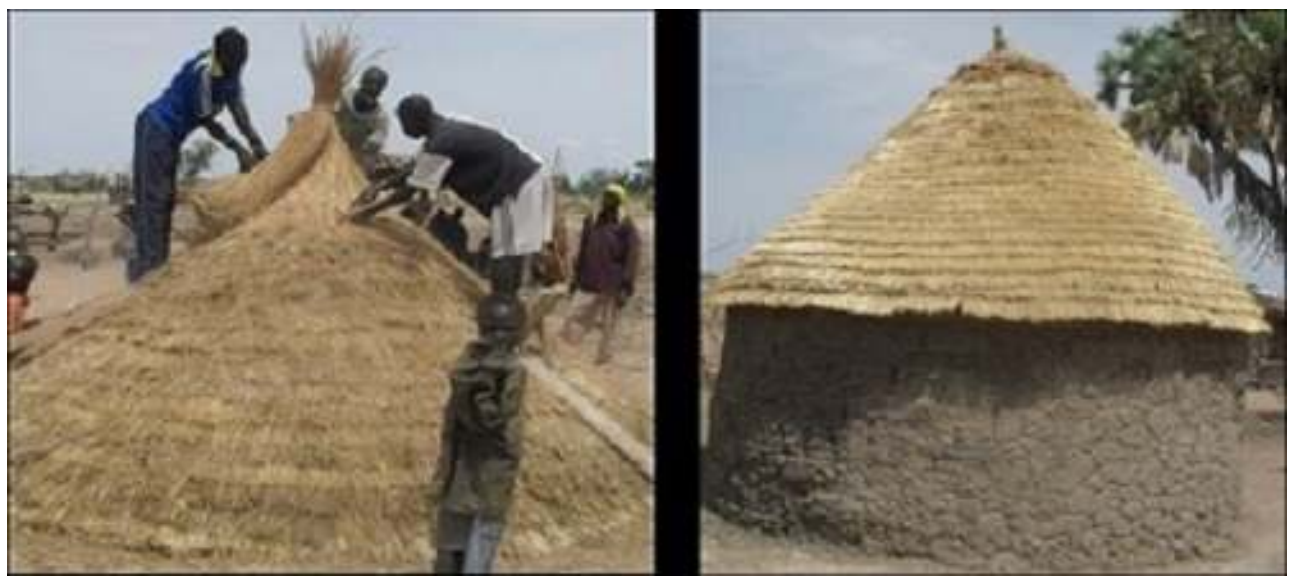

Cliché : Jean Gormo, avril 2013

\section{Construction des greniers}

La région sahélienne dans laquelle vivent les populations toupouri se caractérise par une agriculture céréalière. Face à l'adversité du climat, ces peuples ont construit les greniers dont la principale fonction est de conserver les récoltes de l'année. Il est très rare que les termites et les rongeurs y pénètrent.

De par leur matériau de construction, on distingue en général deux types de greniers:

l'un en paille et l'autre en banco. Néanmoins, les deux présentent la même configuration architecturale que la case ronde.

Le grenier en paille est de forme cylindrique, obtenu à partir de plusieurs séko enroulés. Ces séko sont une sorte de natte végétale tressée à partir des graminées d'Andropogon giganteus (Dousgoum) et Cymbopogon gigantus (Hélé). Cette technique est aussi utilisée dans la confection des toitures de case. Afin de renforcer la résistance du grenier, des piquets sont nécessaires pour maintenir la forme cylindrique. L'intérieur du grenier est alors crépi. L'on utilise le banco mélangé à de la bouse de vache, auquel sont incorporées des plantes possédant des vertus insecticides ou insectifuges.

Le grenier en banco est facile à mettre en œuvre. Le modelage est la technique de transformation. Elle consiste à pétrir l'argile à laquelle on ajoute de la paille. Celle-ci est utilisée comme dégraissant afin d'éviter à la paroi les fendillements au cours du séchage. Elle donne au mur plus de tenu contre les averses. On n'utilise pas directement le torchis ainsi obtenu. Il est laissé à l'air libre pendant environ deux jours avant d'être remouillé. La technique d'élévation de la paroi du grenier consiste en une juxtaposition des couches d'argiles qu'on dispose de manière oblique jusqu'à obtenir la dimension désirée. Le torchis est longuement malaxé, aplani et modelé par la paume des mains avant d'être enroulés en spirales superposées ${ }^{18}$. Joanny Guillard qualifie le grenier en banco appelé Bolé (Toupouri) de :

«type paléo-négritique, est constitué par une énorme jarre à col assez large pour qu'un homme puisse y pénétrer, recouverte en partie de chaume et coiffé de paille, lui donnant l'aspect d'une petite case ronde sur pilotis. ${ }^{19}$. 
41 La couverture de paille est faite à base d'Andropogon tectorum (Ganebo). La base du grenier est obtenue à partir d'un mélange de paille, de branchages et de boue. Le grenier en paille a une paroi semblable au grenier en banco, obtenue à base du bois de Guiera senegalensis ( Furri). Ces bois sont liés entre eux par une corde. Trois bourrelets circulaires soutiennent ces bois. Ces ceintures sont faites à base de Sesbania pachycarpa (Diw). La base de ce grenier est quant à elle couverte essentiellement de paille et de branchages. De par le matériau utilisé pour sa construction, ce grenier est facilement transportable.

Ces deux types de greniers sont soutenus par quatre ou six piliers fourchus obtenus à partir des bois aux bonnes côtes mécaniques et technologiques élevées et qui présentent une bonne durabilité naturelle. Ces caractères sont nécessaires dans la mesure où ces piliers sont directement soumis aux agressions diverses. De ce fait le Prosopis africana ( $\left.W a^{\prime} a\right)$ est très sollicité. À défaut de cette essence, le paysan toupouri utilise Terminalia macroptera (Hobé).

Photo 2. Un grenier en pays toupouri

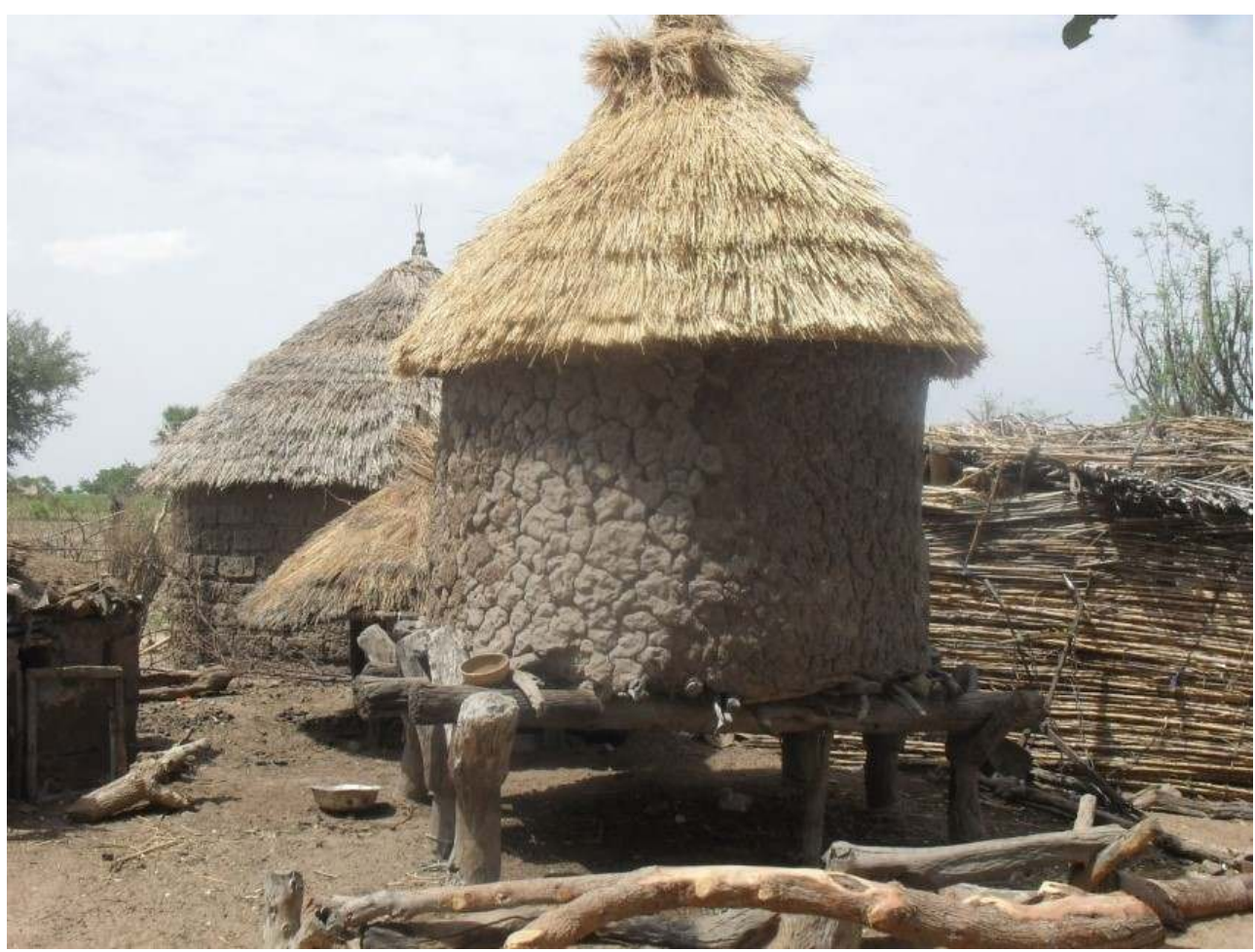

Cliché : Jean Gormo, avril 2013.

\section{Conclusion}

Il s'est agi de montrer dans cet article les différents modes opératoires mis en œuvre par les Toupouri du Nord-Cameroun en vue d'une exploitation efficiente de leurs ressources végétales. Pour ce faire, ils utilisent principalement deux méthodes pour le choix d'une essence végétale. Ces derniers ont en effet grâce à une découverte fortuite, à l'observation ou à l'habitude pu découvrir les qualités d'un arbre. Conscients du fait que l'arbre tout comme l'homme possède un souffle divin, l'abattage des arbres est réglementé par des rituels. Ceux-ci ont pour finalité d'éviter la colère de l'arbre. Pour l'abattage, les 
bûcherons utilisent des haches et des machettes réalisées par la caste des forgerons toupouri.

L'abattage des grands arbres est souvent un travail d'équipe qui dure plusieurs jours. Il requiert une méthode propre et nécessite beaucoup de précision. La première phase consiste à préparer la zone d'abattage. Ces modalités techniques préparatoires achevées, l'équipe d'abattage peut commencer le travail proprement dit. L'orientation de la chute de l'arbre dans la direction choisie n'est pas hasardeuse. Elle tient compte d'un certain nombre de mesures très précises. Ainsi, pour abattre les arbres, les Toupouri se remettaient à la tradition qui prévoyait ce travail en saison sèche.

De par sa polyvalence fonctionnelle, l'arbre constitue le support matériel de plusieurs activités notamment pour l'architecture, le mobilier, les instruments de musique, les outils de chasse, pêche, agriculture et est utilisé comme combustible. Dans le cadre de cet article, l'accent a été mis sur l'architecture qui regroupe la fabrication de la charpente et la construction des greniers. D'une apparence extérieure sobre, la case toupouri révèle une architecture ingénieuse basée sur le travail de la paille. La confection des toitures se fait à l'aide de la paille et des cordes. La toiture a une forme conique, une base ronde et un sommet pointu. La charpente, constituée de bois liés à un ensemble de ceintures circulaires, représente l'ossature de la toiture. Une charpente est généralement faite de sept ceintures circulaires qui vont de la base au sommet. Dans une région où profilèrent termites et insectes xylophages, les bois qui constituent la charpente doivent présenter de bonnes caractéristiques mécaniques.

De par leur matériau de construction, on distingue en général deux types de greniers: l'un en paille et l'autre en banco. Néanmoins les deux présentent la même configuration architecturale que la case ronde. Ces deux types de greniers sont soutenus par quatre ou six piliers fourchus obtenus à partir des bois aux bonnes côtes mécaniques et technologiques élevées et qui présentent une bonne durabilité naturelle. Ce savoir-faire endogène est aussi présent dans d'autres activités et mérite d'être visité.

\section{BIBLIOGRAPHIE}

BARIAUX Daniel, DEMOLIN Didier, « Naissance de la voix d'un tambour à fente chez les Mangbetu. Du geste de l'artisan à celui du musicien et du danseur ", Cahiers d'ethnomusicologie, 8 , 1995. En ligne, consulté le 19 septembre 2014. URL : http://ethnomusicologie.revues.org/1193.

BENOÎT Jean-Pascal, Kirdi au bord du monde. Un médecin lyonnais au Cameroun, Paris, René Julliard, 1957.

BERGERET Anne, RIBOT Jesse C., L'arbre nourricier en pays Sahélien, Paris, Maison des Sciences de l'homme, 1990.

CAMEROON RADIO AND TELEVISION CORPORATION, « Les arbres à tambour du Burundi », présentation, septembre 2001.

GOLLNHOFER O., SALLÉE P., SILLANS R., Art et artisanat tsogho, Paris, ORSTOM, 1975. 
GORMO Jean, Les hommes et les végétaux dans la société toupouri du Nord-Cameroun. Permanences et changements, Mémoire de Maîtrise en Histoire, Université de Ngaoundéré, 1998.

GORMO Jean, L'homme et les plantes dans les sociétés toupouri et massa du Nord-Cameroun du $\mathrm{XIX}^{\mathrm{e}}$ au XX $\mathrm{X}^{\mathrm{e}}$ siècle, Thèse de Doctorat en Histoire, Université de Ngaoundéré, 2005.

GUILLARD Joanny, Golonpoui Nord-Cameroun. Analyse des conditions de modernisation d'un village du Nord-Cameroun, Paris, Mouton \& Co, 1965.

IATI Bergmans, Les pirogues à balancier des îles du sud du Vanuatu : des temps anciens à la période coloniale, Port Vila, Vanuatu Kaljoral Senta, 2012.

LÉVY-BRUHL Lucien, 1927, L'âme primitive, Paris, Presses Universitaires de France, 1927.

MOTTE-FLORAC Élisabeth, «Ethnobotanique, connaissance de l'homme et de son histoire », in CHRÉTIEN Jean-Pierre (dir.), Histoire rurale de l'Afrique des Grands Lacs :guide de recherches, Paris, Karthala, 1983.

NIZESÉTÉ Bienvenue Denis, « Exploitation et mise en valeur traditionnelle du matériau ligneux par les peuples de l'Ouest-Cameroun ", Société d'Anthropologie du Sud-Ouest, 1996, 31, 3-4, p.

135-157.

NIZESÉTÉ Bienvenue Denis, Les hommes et le bois dans les Hauts plateaux de l'Ouest-Cameroun. Des éléments pour l'archéologie, l'histoire et la technologie du bois dans les Grassfields, Thèse de Doctorat, Université de Paris I Panthéon-Sorbonne, 1992.

PARMENTIER J. L, Le grand livre international du Bois, Paris, Fernand Nathan, 1991.

\section{NOTES}

1. MOTTE-FLORAC Élisabeth, "Ethnobotanique, connaissance de l'homme et de son histoire ", in CHRÉTIEN Jean-Pierre (dir.), Histoire rurale de l'Afrique des Grands Lacs : guide de recherches, Paris, Karthala. 1983, p. 54.

2. MOTTE-FLORAC 1983, op. cit., p. 57.

3. Cette crise des essences végétales est le résultat d'une combinaison de facteurs à la fois anthropiques et naturels. Les facteurs anthropiques comprennent le déboisement sauvage pour l'agriculture, l'architecture, le bois d'œuvre, de chauffe et de chauffage. Les causes naturelles concernent le climat qui est presque désertique.

4. Les qualités technologiques d'un bois sont les différentes propriétés qui permettent à l'arbre d'être mis en avant pour promouvoir son usage en concurrence avec d'autres arbres. Il s'agit de sa densité (bois lourd ou léger), sa durabilité (résistance naturelle aux attaques d'insectes et de champignons), sa rectitude, etc... En effet, les qualités technologiques se rapportent à la capacité d'un bois à se prêter à divers usages. On comprend dès lors que la qualité d'un bois est une notion relative et complexe. Cependant, il existe des défauts rédhibitoires comme la «nervosité des bois ", c'est-à-dire la tendance d'un bois à se déformer ou à fendre spontanément "sans raison apparente", pénalise pratiquement tous les usages.

5. NIZESÉTÉ Bienvenue Denis, Les hommes et le bois dans les Hauts plateaux de l'OuestCameroun. Des éléments pour l'archéologie, l'histoire et la technologie du bois dans les Grassfields, Thèse de Doctorat, Université de Paris 1 Panthéon-Sorbonne, 1992, p.75.

6. GORMO Jean, Les hommes et les végétaux dans la société toupouri du Nord-Cameroun. Permanences et changements, Mémoire de Maîtrise en Histoire, Université de Ngaoundéré, 1998, p.29. 
7. BARIAUX Daniel, DEMOLIN Didier, «Naissance de la voix d'un tambour à fente chez les Mangbetu. Du geste de l'artisan à celui du musicien et du danseur ", Cahiers d'ethnomusicologie, 8, 1995. En ligne, consulté le 19 septembre 2014. URL : http://ethnomusicologie.revues.org/1193.

8. GORMO 2005, op.cit.

9. LÉVY-BRUHL Lucien, 1927, L'âme primitive, Paris, Presses Universitaires de France, 1927, p.20.

10. Le maître initiateur dans la société toupouri est représenté par le Wang so'o qui est en fait le responsable du culte rendu aux ancêtres. C'est sous sa supervision que se déroulent tous les rituels qui rythment la vie communautaire. Il est souvent accompagné dans l'exécution de ses tâches par les initiés à la religion traditionnelle.

11. GORMO 2005, op.cit.

12. IATI Bergmans, Les pirogues à balancier des îles du sud du Vanuatu : des temps anciens à la période coloniale, Port Vila, Vanuatu Kaljoral Senta, 2012, p.33-34.

13. GORMO. 2005, op.cit.

14. CAMEROON RADIO AND TELEVISION CORPORATION, «Les arbres à tambour du Burundi », présentation, septembre 2001.

15. NIZESÉTÉ Bienvenue Denis, «Exploitation et mise en valeur traditionnelle du matériau ligneux par les peuples de l'Ouest-Cameroun ", Société d'Anthropologie du Sud-Ouest, 21, 1996

16. Il s'agit d'une institution culturelle qui regroupe les propriétaires des vaches laitières. Ces derniers se retrouvent sous l'ombre d'un grand arbre à l'écart du village pour y faire la cure de lait et mener d'autres activités culturelles.

17. PARMENTIER J. L., Le grand livre international du Bois, Paris, Fernand Nathan, 1991, p.1.

18. BENOÎT Jean-Pascal, 1957, Kirdi au bord du monde. Un médecin lyonnais au Cameroun, Paris, René Julliard, p.89.

19. GUILLARD Joanny, Golonpoui Nord-Cameroun. Analyse des conditions de modernisation d'un village du Nord-Cameroun, Paris, Mouton \& Co, 1965, p.110.

\section{RÉSUMÉS}

Les Toupouri du Nord-Cameroun, dans leur quête perpétuelle de survie ont toujours cherché à domestiquer la nature, à l'humaniser. Dans ce milieu contraignant marqué par l'aridité et par les aléas climatiques importants, les populations ont su adapter leur manière de vivre et leur savoirfaire afin de mieux s'y insérer. L'article analyse non seulement les divers cheminements techniques que suivaient les Toupouri pour exploiter leur couvert végétal, mais aussi s'intéresse aux multiples utilisations et transformations réservées aux arbres. Pour leur besoin en bois d'œuvre et d'architecture, les Toupouri du Nord-Cameroun utilisent principalement deux méthodes pour le choix d'une essence végétale. Ces derniers peuvent grâce à une découverte fortuite, à l'observation ou à l'habitude découvrir les qualités d'un arbre. Au regard de leur polyvalence fonctionnelle, les arbres se sont imposés dans la conscience de ce peuple du NordCameroun comme des forces extra humaines dont l'abattage passe nécessairement par l'observation d'un rituel bien précis. En effet, il existe chez les Toupouri des forêts sacrées qui constituent le lieu par excellence du culte rendu aux ancêtres. La pérennité de ces forêts est garantie par l'existence de nombreux interdits qui entourent la gestion de ces forêts. L'arbre en pays toupouri constitue le support matériel de plusieurs activités notamment pour l'architecture, 
le mobilier, les instruments de musique, les outils de chasse, pêche, agriculture et est utilisé comme combustible

In a bid to survive, the Tupuri people of Far North Cameroon, have always sought to tame nature in order to humanize it. In this difficult environment marked by aridity and important climatic changes, they have adapted their way of life and their know-how to better fit in. This paper first analyses the various technical pathways that the Tupuri have followed to exploit their vegetation; secondly, it focuses on the multiple uses and transformations reserved for trees. In order to fulfil their need for timber and architecture, the Tupuri of Far North Cameroon mainly use two methods for the choice of a tree. Through a fortuitous discovery, observation or habit, the qualities of a tree can be discovered. Thanks to their functional usefulness, the trees have been imposed in the consciousness of this people of Far North Cameroon as extra human forces and cutting them necessarily requests the observation of a specific ritual. Indeed, there are sacred forests among the Tupuri that constitute the place par excellence of cults rendered to the ancestors. The sustainability of these forests is guaranteed by the existence of many bans that surround their management. The tree in the Tupuri country constitutes the material support of several activities, particularly for architecture, furniture, musical instruments, hunting tools, fishing, agriculture and is used as fuel.

\section{INDEX}

Mots-clés : histoire des techniques, Afrique, XIXe siècle, XXe siècle, Cameroun, forêt, savoirs écologiques traditionnels

Keywords : history of technology, Africa, XIXth century, XXth century, North Cameroon, traditional ecological knowledge (TEK), forestry

Index géographique : Afrique, Cameroun

Index chronologique : Époque contemporaine

\section{AUTEUR}

\section{JEAN GORMO}

Jean GORMO est membre de plusieurs Centres de recherche : Centre d'Études Africaines de l'Université de Porto au Portugal (CEAUP), Réseau Universitaire des Chercheurs en Histoire Environnementale en France (RUCHE). Il enseigne au Département d'Histoire de la Faculté des Arts, Lettres et Sciences Humaines de l'Université de Maroua au Cameroun. Il est depuis mars 2018, Chef de Département des Sciences Historiques, Archéologiques et du Patrimoine de l'Université de Maroua. Son champ d'investigation est l'histoire environnementale. Deux ouvrages publiés : Maroua Histoire du reboisement d'une ville sahélienne, en 2014 et Des arbres et des hommes. Une contribution à l'histoire des filières arboricoles à Maroua, Cameroun, en 2015. 